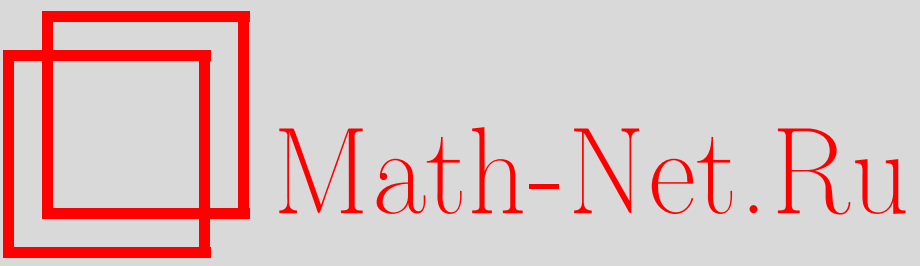

Л. Аккарди, И. В. Волович, С. В. Козырев, Стохастическое приближение в модели взаимодействия частицы с квантовым полем и новая алгебра, ТМФ, 1998, том 116, номер 3, 401-416

DOI: https://doi.org/10.4213/tmf912

Использование Общероссийского математического портала Math-Net.Ru подразумевает, что вы прочитали и согласны с пользовательским соглашением

http://www. mathnet.ru/rus/agreement

Параметры загрузки:

IP : 3.81 .55 .215

26 апреля 2023 г., 11:10:39 
ТЕОРЕТИЧЕСКАЯ

И МАТЕМАТИЧЕСКАЯ

ФИЗИКА

Том 116, № 3

сентябрь, 1998

(C) 1998 г. Л. Аккарди*, И.В. Волович ${ }^{\dagger}$, С. В. Козырев ${ }^{\ddagger}$

\section{СТОХАСТИЧЕСКОЕ ПРИБЛИЖЕНИЕ В МОДЕЛИ ВЗАИМОДЕЙСТВИЯ ЧАСТИЦЫ С КВАНТОВЫМ ПОЛЕМ И НОВАЯ АЛГЕБРА}

Рассмотрено применение стохастического приближения для вычисления корреляционных функций в модели взаимодействия частицы с квантовым полем. Показано, что при этом возникает новая алгебра с перестановочными соотношениями, зависящими от температуры. Эта алгебра представляет собой обобщение свободной (больцмановской) алгебры.

\section{1. ВВЕДЕНИЕ}

В работе рассмотрена модель взаимодействия частицы с квантовым полем. Модели такого типа широко изучались в теории элементарных частиц, в физике твердого тела, в квантовой оптике и других областях [1-4].

В данной работе мы исследуем корреляционные функции модели. При этом используется метод стохастического приближения, который берет начало в работах Ван Хова и Фридрихса. В работах Аккарди, Фриджерио и Лю этот метод был разработан применительно к моделям квантовой оптики [5]. Детальный анализ одной из таких моделей в дипольном приближении был проведен в статье [6]. Суть метода заключается в использовании своеобразного скейлингового предела, когда рассматривается асимптотическое поведение корреляционных функций при больших временах и малых константах связи. При этом для ряда задач предельная динамика оказывается в некотором смысле интегрируемой и удается получить явные выражения для корреляционных функций [6]. Предел называется стохастическим, поскольку свободные корреляторы в этом пределе “ $\delta$-коррелируют” по времени (т.е. имеет место случайный процесс, называемый белым шумом).

Основной результат данной работы заключается в том, что показано, как в температурном стохастическом пределе для модели взаимодействия частицы с квантовым полем возникает новая математическая структура, которую мы будем называть температурной свободной (больцмановской) алгеброй.

\footnotetext{
* Centro Vito Volterra, Universita di Roma 2 di Tor Vergata, Italy

$\dagger$ Математический институт им. В. А. Стеклова РАН, Москва, Россия

${ }^{\ddagger}$ Институт химической физики им. Н. Н. Семенова РАН, Москва, Россия
} 
В работе рассмотриваются корреляционные функции для операторов специального вида (так называемых коллективных переменных). Показано, что в стохастическом пределе происходят упрошения, в результате чего предельная теория описывается температурной больцмановской алгеброй. Это означает, что стохастические пределы корреляторов могут быть воспроизведены некоторым состоянием на температурной больцмановской алгебре. Дальнейшее изучение этой алгебры представляет самостоятельный интерес и должно составлять основу для рассмотрения предельной динамики.

Стохастический предел для данной модели при нулевой температуре был рассмотрен в работах [7-9].

Больцмановская алгебра в простейшем случае описывается соотношениями

$$
B(k) B^{\dagger}\left(k^{\prime}\right)=\delta\left(k-k^{\prime}\right), \quad k, k^{\prime} \in R^{d}
$$

Соотношения такого типа изучались с математической точки зрения в работах [10-15]. В статье [7] подобные соотношения были получены в стохастическом пределе для модели взаимодействия частицы с квантовым полем, а в статье [16] - в пределе больших $N$ для квантовой хромодинамики с калибровочной группой $S U(N)$.

Температурную больцмановскую алгебру, полученную ниже, можно схематически описать как алгебру с образующими $b(k), b^{\dagger}(k), \mathbf{p}$, удовлетворяющими соотношениям

$$
\begin{aligned}
b(k) b^{\dagger}\left(k^{\prime}\right) & =\frac{\delta(\widetilde{\omega}(k)+k \mathbf{p})}{1-e^{-\beta \omega(k)}} \delta\left(k-k^{\prime}\right), \\
b(k) \mathbf{p} & =(\mathbf{p}+k) b(k) .
\end{aligned}
$$

Здесь $\omega(k)$ - одночастичная энергия квантового поля, $\beta$ - обратная температура. Эту алгебру можно рассматривать как своеобразную деформацию больцмановской алгебры.

В разделе 2 дается обшее описание стохастического предела. В разделе 3 формулируется теорема 1 о стохастическом пределе и рассматривается пример вычисления 2и 4-точечных корреляционных функций. В разделе 4 доказывается теорема 2, даюшая описание температурной свободной алгебры. Полное доказательство теоремы 1 приведено в разделе 5 .

\section{2. СТОХАСТИЧЕСКИЙ ПРЕДЕЛ}

Стохастический предел есть своеобразный скейлинговый предел в квантовой теории. Рассмотрим систему, описываемую гамильтонианом

$$
H=H_{0}+\lambda H_{\mathrm{I}} .
$$

Рассмотрим оператор эволющии $U_{t}^{(\lambda)}=e^{i t H_{0}} e^{-i t H}$, удовлетворяюший уравнению Шредингера в представлении взаимодействия:

$$
\frac{\partial}{\partial t} U_{t}^{(\lambda)}=-i \lambda H_{\mathrm{I}}(t) U_{t}^{(\lambda)}, \quad U_{0}^{(\lambda)}=1,
$$


где $H_{\mathrm{I}}(t)=e^{i t H_{0}} H_{\mathrm{I}} e^{-i t H_{0}}$. Здесь $\lambda$ мы считаем малой постоянной и будем изучать эффект накапливаюшихся за долгое время малых возмушений. Для этого осушествим предельные переходы $\lambda \rightarrow 0, t \rightarrow \infty$ и сделаем в эволюционном уравнении замену $t \rightarrow t / \lambda^{2}$ :

$$
\frac{\partial}{\partial t} U_{t / \lambda^{2}}^{(\lambda)}=-\frac{i}{\lambda} H_{\mathrm{I}}\left(\frac{t}{\lambda^{2}}\right) U_{t / \lambda^{2}}^{(\lambda)} .
$$

Естественно предположить, что если существуют пределы

$$
\begin{aligned}
\lim _{\lambda \rightarrow 0} U_{t / \lambda^{2}}^{(\lambda)} & =U_{t}, \\
\lim _{\lambda \rightarrow 0} \frac{1}{\lambda} H_{\mathrm{I}}\left(\frac{t}{\lambda^{2}}\right) & =H_{t},
\end{aligned}
$$

то предельный оператор $U_{t}$ удовлетворяет уравнению

$$
\partial_{t} U_{t}=-i H_{t} U_{t}, \quad U_{0}=1 .
$$

Пределы понимаются в смысле сходимости корреляторов. При указанном предельном переходе многие задачи становятся точно решаемыми. Замена $t \rightarrow t / \lambda^{2}$ и устремление $\lambda$ к нулю соответствует одновременному пределу $\lambda \rightarrow 0, t \rightarrow \infty$ при $\lambda^{2} t=$ const. Здесь $\lambda^{2} t$ интерпретируется как "медленная" шкала времени. Этот предел описывает главные вклады в динамику в режиме слабой связи на больших временах, т.е. эффект накапливаюшихся слабых возмушений. Физическая идея состоит в том, что в “медленной” шкале времени квантовое поле выглядит хаотическим объектом, квантовым белым шумом, т.е. $\delta(t)$-коррелированным квантовым случайным процессом $b(t, k)$, называемым также “мастер-полем". Предметом данной работы является нахождение перестановочных соотношений “мастер-поля".

Мы будем считать, что квантово-механическая система есть тройка объектов (алгебра наблюдаемых $\mathcal{A}$, пространство состояний, оператор эволюции). Более того, мы будем считать, что пространство состояний есть гильбертово пространство представления Гельфанда-Наймарка-Сигала (ГНС), порожденного некоторым состоянием $\langle\cdot\rangle$ на алгебре наблюдаемых. В качестве такого функционала можно взять, например, вакуумное либо температурное среднее. В качестве оператора эволюции надо взять введенный выше оператор эволюции в представлении взаимодействия $U_{t}^{(\lambda)}$.

Стохастический предел алгебры наблюдаемых строится следующим образом. Элементу $A$ алгебры наблюдаемых поставим в соответствие его свободную эволюцию $A(t)$ $=e^{i t H_{0}} A e^{-i t H_{0}}$. Подберем элементы $A_{i}$, для которых существуют нетривиальные пределы выражений вида

$$
\lim _{\lambda \rightarrow 0}\left\langle\frac{1}{\lambda} A_{1}\left(\frac{t_{1}}{\lambda^{2}}\right) \ldots \frac{1}{\lambda} A_{k}\left(\frac{t_{k}}{\lambda^{2}}\right)\right\rangle .
$$

Попробуем найти алгебру $\mathcal{B}$ (элементы которой будем обозначать $B_{i}$ ) и состояние $\langle\langle\cdot\rangle\rangle$ на ней таким образом, чтобы выполнялось равенство

$$
\lim _{\lambda \rightarrow 0}\left\langle\frac{1}{\lambda} A_{1}\left(\frac{t_{1}}{\lambda^{2}}\right) \ldots \frac{1}{\lambda} A_{k}\left(\frac{t_{k}}{\lambda^{2}}\right)\right\rangle=\left\langle\left\langle B_{1}\left(t_{1}\right) \ldots B_{k}\left(t_{k}\right)\right\rangle\right\rangle
$$


Полученную алгебру $\mathcal{B}$ будем называть стохастическим пределом алгебры наблюдаемых $\mathcal{A}$. ГНС-представление этой алгебры порождено состоянием $\langle\langle\cdot\rangle\rangle$. Фактически для исследования эволюции, задаваемой уравнением (1), достаточно вычислить стохастический предел для элементов алгебры наблюдаемых, входяших в гамильтониан взаимодействия $H_{\mathrm{I}}$.

Анализ ряда теории возмущений (см. ниже) позволяет сделать следующий вывод. Используя теорему Вика, можно представить ряд теории возмущений после нормального упорядочения в виде ряда по диаграммам. Для некоторых моделей в стохастическом пределе "выживают" только полупланарные диаграммы. Полупланарным диаграммам соответствует алгебра свободных операторов рождения-уничтожения. Для рассматриваемой модели будет установлено, что в стохастическом пределе получается деформация больцмановской алгебры, называемая температурной свободной алгеброй.

\section{3. МОДЕЛЬ ЧАСТИЦЫ, ВЗАИМОДЕЙСТВУЮЩЕЙ С КВАНТОВЫМ ПОЛЕМ}

Мы будем изучать модель частицы, взаимодействующей с квантовым полем при ненулевой температуре. Операторы координаты и импульса квантовой частицы $q=$ $\left(q_{1}, \ldots, q_{d}\right)$ и $p=\left(p_{1}, \ldots, p_{d}\right)$ удовлетворяют перестановочным соотношениям

$$
\left[q_{m}, p_{n}\right]=i \delta_{m n}
$$

Квантовое поле описывается бозонными операторами (операторнозначными обобщенными функциями)

$$
a(k)=\left(a_{1}(k), \ldots, a_{d}(k)\right), \quad a^{\dagger}(k)=\left(a_{1}^{\dagger}(k), \ldots, a_{d}^{\dagger}(k)\right), \quad k \in R^{d},
$$

с перестановочными соотношениями

$$
\left[a_{j}(k), a_{h}^{\dagger}\left(k^{\prime}\right)\right]=\delta_{j h} \delta\left(k-k^{\prime}\right) .
$$

Гамильтониан рассматриваемой системы имеет вид

$$
H=H_{0}+\lambda H_{\mathrm{I}}=\int \omega(k) a^{\dagger}(k) a(k) d k+\frac{1}{2} p^{2}+\lambda H_{\mathrm{I}},
$$

где $\omega$ - некоторая положительная функция на $R^{d}$, реалистичный пример которой есть $\omega(k)=|k|, H_{\mathrm{I}}$ задает взаимодействие свободной частищы с квантовым полем. Взаимодействие частицы с полем выражено через потенциал $\mathcal{A}(x)$, описывающий квантовое поле в точке пространства $x \in R^{d}: \mathcal{A}(x)$ есть потенциал поля, действуюшего на частицу с координатой $x$. Явная форма гамильтониана взаимодействия имеет вид

$$
H_{\mathrm{I}}=p \mathcal{A}(q)+\mathcal{A}(q) p,
$$

где

$$
\mathcal{A}(q)=\int d k\left\{g(k) e^{i k \cdot q} a^{\dagger}(k)+\bar{g}(k) e^{-i k \cdot q} a(k)\right\}
$$


Временная зависимость $H_{\mathrm{I}}(t)$ задается через оператор

$$
a_{\lambda}(t, k)=\frac{1}{\lambda} e^{i \frac{t}{\lambda^{2}} H_{0}} e^{-i k q} a(k) e^{-i \frac{t}{\lambda^{2}} H_{0}}=\frac{1}{\lambda} e^{\frac{-i(\tilde{\omega}(k)+k p) t}{\lambda^{2}}} e^{-i k q} a(k),
$$

где $\widetilde{\omega}(k)=\omega(k)+k^{2} / 2$.

Мы будем изучать предел температурных корреляционных функций

$$
\lim _{\lambda \rightarrow 0}\left\langle a_{\lambda}^{\epsilon_{N}}\left(t_{N}, k_{N}\right) a_{\lambda}^{\epsilon_{N-1}}\left(t_{N-1}, k_{N-1}\right) \ldots a_{\lambda}^{\epsilon_{1}}\left(t_{1}, k_{1}\right)\right\rangle .
$$

Средние

$$
\begin{aligned}
\left\langle a(k) a^{\dagger}\left(k^{\prime}\right)\right\rangle & =\frac{\delta\left(k-k^{\prime}\right)}{1-e^{-\beta \omega(k)}}, \\
\left\langle a^{\dagger}\left(k^{\prime}\right) a(k)\right\rangle & =\frac{\delta\left(k-k^{\prime}\right)}{e^{\beta \omega(k)}-1}
\end{aligned}
$$

- это температурные бозонные корреляторы (остальные вычисляются по температурной теореме Вика), $\varepsilon \equiv\left\{\epsilon_{N}, \ldots, \epsilon_{1}\right\} \in\{1,0\}^{N}, \epsilon \in\{1,0\} \quad\left(\epsilon=0\right.$ для $a$ и $\epsilon=1$ для $\left.a^{\dagger}\right)$. Температурное среднее $\langle\cdot\rangle$ понимается как гиббсовское среднее по степеням свободы поля, не затрагивающее степеней свободы квантовой частицы, т.е.

$$
\langle X\rangle=\frac{\operatorname{tr}\left(X \exp \left\{-\beta \int \omega(k) a^{\dagger}(k) a(k) d k\right\}\right)}{\operatorname{tr}\left(\exp \left\{-\beta \int \omega(k) a^{\dagger}(k) a(k) d k\right\}\right)} .
$$

По степеням свободы квантовой частищы среднее $\langle\cdot\rangle$ действует как условное ожидание, т.е. $\langle p X\rangle=p\langle X\rangle$.

При $N=2 n$ и равенстве числа операторов рождения и уничтожения рассмотрим разбиение $\sigma(\varepsilon)$ последовательности $\varepsilon$ на пары из 0 и 1 , что отвечает виковскому разложению

$$
\left\langle a_{\lambda}^{\epsilon_{N}}\left(t_{N}, k_{N}\right) a_{\lambda}^{\epsilon_{N-1}}\left(t_{N-1}, k_{N-1}\right) \ldots a_{\lambda}^{\epsilon_{1}}\left(t_{1}, k_{1}\right)\right\rangle
$$

на пары операторов рождения-уничтожения. Произвольное разбиение такого вида соответствует некоторой виковской диаграмме. Нас будут интересовать разбиения, соответствуюшие полупланарным непересекаюшимся диаграммам. Такие разбиения будем называть нетривиальными.

Виковская диаграмма по разбиению строится следуюшим образом. Расположим индексы 0 и 1 последовательности $\varepsilon$ на отрезке в порядке возрастания номера. Соединим дугами индексы, для которых присутствует пара в разбиении $\sigma(\varepsilon)$. Если все дуги полученной диаграммы расположить в одной полуплоскости относительно отрезка, причем дуги не пересекаются, то такую диаграмму назовем полуплоской непересекающейся, а соответствуюшее разбиение $\sigma(\varepsilon)$ - нетривиальным. 
ТЕОрема 1. Предел температурных коррелячионных функций всегда существует. Кроме того,

1) если число операторов рождения не равно числу операторов уничтожсения, то коррелятор равен нулю (даже до предельного перехода),

2) если число операторов рождения равно числу операторов уничтожения, то предел коррелятора

$$
\lim _{\lambda \rightarrow 0}\left\langle a_{\lambda}^{\epsilon_{2 n}}\left(t_{2 n}, k_{2 n}\right) a_{\lambda}^{\epsilon_{2 n-1}}\left(t_{2 n-1}, k_{2 n-1}\right) \ldots a_{\lambda}^{\epsilon_{1}}\left(t_{1}, k_{1}\right)\right\rangle
$$

представляется в виде суммы по нетривиальным разбиениям

$$
\begin{aligned}
\sum_{\sigma(\varepsilon)} & \prod_{h=1}^{n} \delta\left(k_{m_{h}^{\prime}}-k_{m_{h}}\right) c_{m_{h} m_{h}^{\prime}}\left(k_{m_{h}}\right) 2 \pi \delta\left(t_{m_{h}^{\prime}}-t_{m_{h}}\right) \times \\
& \times \delta\left(\widetilde{\omega}\left(k_{m_{h}}\right)+k_{m_{h}} p+\sum_{\alpha}(-1)^{\varepsilon_{\alpha}} \chi_{\left(m_{\alpha}, m^{\prime}{ }_{\alpha}\right)}\left(m_{h}\right) k_{m_{\alpha}} k_{m_{h}}-\varepsilon_{h} k_{m_{h}}^{2}\right),
\end{aligned}
$$

әде $\left\{\left(m^{\prime}{ }_{j}, m_{j}\right) ; j=1, \ldots, n\right\}-$ разбиения $\{1, \ldots, 2 n\}$, соответствующие разбиениям $\sigma(\varepsilon)$.Величина $m_{h}^{\prime}$ соответствует оператору уничтожения, $m_{h}$ - оператору рожсдения,

$$
\begin{array}{ll}
c_{m_{h} m_{h}^{\prime}}(k)=\frac{1}{1-e^{-\beta \omega(k)}}, & m_{h}^{\prime}>m_{h}, \\
c_{m_{h} m_{h}^{\prime}}(k)=\frac{1}{e^{\beta \omega(k)}-1}, & m_{h}^{\prime}<m_{h},
\end{array}
$$

$(-1)^{\varepsilon_{h}}=1$ для $m_{h}^{\prime}>m_{h} u(-1)^{\varepsilon_{h}}=-1$ для $m_{h}^{\prime}<m_{h}$. Функиия $\chi_{\left(m_{\alpha}, m_{\alpha}^{\prime}\right)}$ есть индикатор (характеристическая функиия) отрезка $\left(m_{\alpha}, m^{\prime}{ }_{\alpha}\right)$, т.е. суммирование по $\alpha$ ведется по парам $\left(m_{\alpha}, m_{\alpha}^{\prime}\right)$ таким, что $m_{h}$ расположен между $m_{\alpha} u m_{\alpha}^{\prime}$.

Доказательство теоремы 1 будет проведено в разделе 5. Здесь для иллюстрации теоремы 1 будут вычислены 2-точечные и 4-точечные корреляторы.

Вычислим 2-точечный коррелятор

$$
\begin{aligned}
& \lim _{\lambda \rightarrow 0}\left\langle a_{\lambda}\left(t, k_{1}\right) a_{\lambda}^{\dagger}\left(\tau, k_{2}\right)\right\rangle= \\
& \quad=\lim _{\lambda \rightarrow 0} \frac{1}{\lambda^{2}} e^{-i t / \lambda^{2}\left(\widetilde{\omega}\left(k_{1}\right)+k_{1} p\right)} e^{-i q\left(k_{1}-k_{2}\right)} e^{i \frac{\tau}{\lambda^{2}}\left(\widetilde{\omega}\left(k_{2}\right)+k_{2} p\right)}\left\langle a\left(k_{1}\right) a^{\dagger}\left(k_{2}\right)\right\rangle .
\end{aligned}
$$

Используя формулы для бозонных 2-точечных корреляторов, получим

$$
\lim _{\lambda \rightarrow 0} \frac{1}{\lambda^{2}} e^{-i \frac{t-\tau}{\lambda^{2}}\left(\widetilde{\omega}\left(k_{1}\right)+k_{1} p\right)} \frac{\delta\left(k_{1}-k_{2}\right)}{1-e^{-\beta \omega\left(k_{1}\right)}} .
$$

Применив формулу

$$
\lim _{\lambda \rightarrow 0} \frac{1}{\lambda^{2}} e^{\frac{i t}{\lambda^{2}}(\widetilde{\omega}(k)+k p)}=2 \pi \delta(\widetilde{\omega}(k)+k p) \delta(t)
$$


получим для предела 2-точечного коррелятора выражение

$$
\lim _{\lambda \rightarrow 0}\left\langle a_{\lambda}\left(t, k_{1}\right) a_{\lambda}^{\dagger}\left(\tau, k_{2}\right)\right\rangle=2 \pi \delta(t-\tau) \delta\left(\widetilde{\omega}\left(k_{1}\right)+k_{1} p\right) \frac{\delta\left(k_{1}-k_{2}\right)}{1-e^{-\beta \omega\left(k_{1}\right)}} .
$$

Рассмотрим теперь 2-точечный коррелятор

$$
\begin{aligned}
& \lim _{\lambda \rightarrow 0}\left\langle a_{\lambda}^{\dagger}\left(\tau, k_{2}\right) a_{\lambda}\left(t, k_{1}\right)\right\rangle= \\
& \quad=\lim _{\lambda \rightarrow 0} \frac{1}{\lambda^{2}}\left\langle a^{\dagger}\left(k_{2}\right) e^{i k_{2} q} e^{i \frac{\tau}{\lambda^{2}}\left(\widetilde{\omega}\left(k_{2}\right)+k_{2} p\right)} e^{-i \frac{t}{\lambda^{2}}\left(\widetilde{\omega}\left(k_{1}\right)+k_{1} p\right)} e^{-i k_{1} q} a\left(k_{1}\right)\right\rangle .
\end{aligned}
$$

Используя перестановочные соотношения для вейлевских операторов

$$
e^{i \alpha p} e^{i \beta q}=e^{i \beta q} e^{i \alpha p} e^{i \alpha \beta}
$$

где $[p, q]=-i$, получим для коррелятора (13) следующее выражение:

$$
\lim _{\lambda \rightarrow 0} \frac{1}{\lambda^{2}} \frac{\delta\left(k_{2}-k_{1}\right)}{e^{\beta \omega\left(k_{1}\right)}-1} e^{-i \frac{t-\tau}{\lambda^{2}}\left(\widetilde{\omega}\left(k_{1}\right)+k_{1} p-k_{1}^{2}\right)} .
$$

Применив опять формулу (11), найдем, что

$$
\lim _{\lambda \rightarrow 0}\left\langle a_{\lambda}^{\dagger}\left(\tau, k_{2}\right) a_{\lambda}\left(t, k_{1}\right)\right\rangle=2 \pi \delta(t-\tau) \delta\left(\widetilde{\omega}\left(k_{1}\right)+k_{1} p-k_{1}^{2}\right) \frac{\delta\left(k_{2}-k_{1}\right)}{e^{\beta \omega\left(k_{1}\right)}-1} .
$$

Вычислим предел 4-точечного коррелятора

$$
\lim _{\lambda \rightarrow 0}\left\langle a_{\lambda}\left(t_{1}, k_{1}\right) a_{\lambda}\left(t_{2}, k_{2}\right) a_{\lambda}^{\dagger}\left(t_{2}^{\prime}, k_{2}^{\prime}\right) a_{\lambda}^{\dagger}\left(t_{1}^{\prime}, k_{1}^{\prime}\right)\right\rangle .
$$

По температурной теореме Вика имеем

$$
\begin{aligned}
\left\langle a\left(k_{1}\right) a\left(k_{2}\right) a^{\dagger}\left(k_{2}^{\prime}\right) a^{\dagger}\left(k_{1}^{\prime}\right)\right\rangle= & \frac{1}{1-e^{-\beta \omega\left(k_{1}\right)}} \frac{1}{1-e^{-\beta \omega\left(k_{2}\right)}} \times \\
& \times\left(\delta\left(k_{2}-k_{2}^{\prime}\right) \delta\left(k_{1}-k_{1}^{\prime}\right)+\delta\left(k_{1}-k_{2}^{\prime}\right) \delta\left(k_{2}-k_{1}^{\prime}\right)\right) .
\end{aligned}
$$

Формула (16) для бозонного коррелятора $\left\langle a\left(k_{1}\right) a\left(k_{2}\right) a^{\dagger}\left(k_{2}^{\prime}\right) a^{\dagger}\left(k_{1}^{\prime}\right)\right\rangle$ содержит два члена, пропорциональных $\delta$-функциям, которые соответствуют виковским диаграммам. Вычислим первый член $\mathcal{L}_{1}$, пропорциональный $\delta\left(k_{1}-k_{1}^{\prime}\right) \delta\left(k_{2}-k_{2}^{\prime}\right)$. Имеем

$$
\begin{aligned}
\mathcal{L}_{1}= & \lim _{\lambda \rightarrow 0} \frac{1}{1-e^{-\beta \omega\left(k_{1}\right)}} \frac{1}{1-e^{-\beta \omega\left(k_{2}\right)}} \delta\left(k_{1}-k_{1}^{\prime}\right) \delta\left(k_{2}-k_{2}^{\prime}\right) \times \\
& \times \frac{1}{\lambda^{4}} e^{-i \frac{t_{1}-t_{1}^{\prime}}{\lambda^{2}}\left(\widetilde{\omega}\left(k_{1}\right)+k_{1} p\right)} e^{-i \frac{t_{2}-t_{2}^{\prime}}{\lambda^{2}}\left(\widetilde{\omega}\left(k_{2}\right)+k_{2} p\right)} e^{-i \frac{t_{2}-t_{2}^{\prime}}{\lambda^{2}} k_{1} k_{2}} .
\end{aligned}
$$

Из формулы (11) получаем, что

$$
\begin{aligned}
\mathcal{L}_{1}= & (2 \pi)^{2} \frac{1}{1-e^{-\beta \omega\left(k_{1}\right)}} \frac{1}{1-e^{-\beta \omega\left(k_{2}\right)}} \times \\
& \times \delta\left(k_{1}-k_{1}^{\prime}\right) \delta\left(k_{2}-k_{2}^{\prime}\right) \delta\left(t_{1}-t_{1}^{\prime}\right) \delta\left(t_{2}-t_{2}^{\prime}\right) \times \\
& \times \delta\left(\widetilde{\omega}\left(k_{1}\right)+k_{1} p\right) \delta\left(\widetilde{\omega}\left(k_{2}\right)+k_{2} p+k_{1} k_{2}\right) .
\end{aligned}
$$

Вычислим второй член $\mathcal{L}_{2}$ коррелятора, пропорциональный $\delta\left(k_{1}-k_{2}^{\prime}\right) \delta\left(k_{2}-k_{1}^{\prime}\right)$. По формуле (11) находим

$$
\begin{aligned}
\mathcal{L}_{2}= & \lim _{\lambda \rightarrow 0} \frac{1}{1-e^{-\beta \omega\left(k_{1}\right)}} \frac{1}{1-e^{-\beta \omega\left(k_{2}\right)}} \delta\left(k_{1}-k_{2}^{\prime}\right) \delta\left(k_{2}-k_{1}^{\prime}\right) \times \\
& \times \frac{1}{\lambda^{4}} e^{-i \frac{t_{1}-t_{2}^{\prime}}{\lambda^{2}}\left(\widetilde{\omega}\left(k_{1}\right)+k_{1} p\right)} e^{-i \frac{t_{2}-t_{1}^{\prime}}{\lambda^{2}}\left(\widetilde{\omega}\left(k_{2}\right)+k_{2} p\right)} e^{-i \frac{t_{2}-t_{2}^{\prime}}{\lambda^{2}} k_{1} k_{2}}=0 .
\end{aligned}
$$

Таким образом, 4-точечный коррелятор задается формулой (18). 


\section{4. ТЕМПЕРАТУРНАЯ СВОБОДНАЯ АЛГЕБРА}

Нашей целью является построение алгебры $\mathcal{B}$ и состояния $\langle\langle\cdot\rangle\rangle$ на этой алгебре. При этом корреляционные функции алгебры $\mathcal{B}$ относительно состояния $\langle\langle\cdot\rangle\rangle$ должны воспроизводить стохастические пределы температурных корреляторов алгебры наблюдаемых $\mathcal{A}$, т.е. должны выполняться тождества

$$
\begin{array}{r}
\lim _{\lambda \rightarrow 0}\left\langle a_{\lambda}^{\epsilon_{N}}\left(t_{N}, k_{N}\right) a_{\lambda}^{\epsilon_{N-1}}\left(t_{N-1}, k_{N-1}\right) \ldots a_{\lambda}^{\epsilon_{1}}\left(t_{1}, k_{1}\right)\right\rangle= \\
=\left\langle\left\langle b^{\epsilon_{N}}\left(t_{N}, k_{N}\right) b^{\epsilon_{N-1}}\left(t_{N-1}, k_{N-1}\right) \ldots b^{\epsilon_{1}}\left(t_{1}, k_{1}\right)\right\rangle\right\rangle .
\end{array}
$$

Коррелятор, полученный в теореме 1 , можно описать следуюшим образом. Коррелятор, соответствуюший последовательности индексов $\varepsilon$, равен сумме по полупланарным диаграммам, соответствуюшим разбиениям $\sigma(\varepsilon)$. Вклад от каждой полупланарной диаграммы равен произведению вкладов по дугам. Вклад от дуги состоит из $\delta$-функции по импульсам с температурным весом (что равно вкладу в бозонньй коррелятор), $2 \pi \delta$-функции по соответствуюшим временам и $\delta$-функции от фазы. В фазу входит член, зависяший от самой дуги и сумма вкладов от дуг, находящихся внутри данной. Такая простая структура коррелятора позволяет выразить его как состояние на алгебре $\mathcal{B}$, структура которой задается следующей теоремой.

ТЕОРема 2. Корреляторы из теоремы 1 воспроизводятся, если величину $b(t, k)$ взять в виде суммы двух свободно независимых случайных величин

$$
b(t, k)=b_{1}(t, k)+b_{2}^{\dagger}(t, k),
$$

удовлетворяющих соотношениям температурной свободной алгебры, и выбрать функиионал $\langle\langle\cdot\rangle\rangle$ равным вакуумному среднему для полей $b_{i}(t, k)$. Температурная свободная алгебра имеет образующие $\left\{p, b_{i}(t, k), b_{i}^{\dagger}(t, k), i=1,2\right\}$, удовлетворяющие соотношениям

$$
\begin{aligned}
b_{1}\left(t, k_{1}\right) b_{1}^{\dagger}\left(\tau, k_{2}\right) & =2 \pi \delta(t-\tau) \delta\left(\widetilde{\omega}\left(k_{1}\right)+k_{1} p\right) \frac{\delta\left(k_{1}-k_{2}\right)}{1-e^{-\beta \omega\left(k_{1}\right)}}, \\
b_{2}\left(t, k_{1}\right) b_{2}^{\dagger}\left(\tau, k_{2}\right) & =2 \pi \delta(t-\tau) \delta\left(\widetilde{\omega}\left(k_{1}\right)+k_{1}\left(p-k_{1}\right)\right) \frac{\delta\left(k_{1}-k_{2}\right)}{e^{\beta \omega\left(k_{1}\right)}-1}, \\
b_{1}\left(t, k_{1}\right) b_{2}^{\dagger}\left(\tau, k_{2}\right) & =b_{2}\left(\tau, k_{2}\right) b_{1}^{\dagger}\left(t, k_{1}\right)=0, \\
b_{1}(t, k) p & =(p+k) b_{1}(t, k), \\
b_{2}(t, k) p & =(p-k) b_{2}(t, k) .
\end{aligned}
$$

ДоКАЗАТЕЛЬСтво представляет собой прямое вычисление. Разложим коррелятор

$$
\left\langle\left\langle b^{\epsilon_{N}}\left(t_{N}, k_{N}\right) b^{\epsilon_{N-1}}\left(t_{N-1}, k_{N-1}\right) \ldots b^{\epsilon_{1}}\left(t_{1}, k_{1}\right)\right\rangle\right\rangle
$$

в сумму корреляторов мономов от образуюших $b_{i}^{\epsilon}(t, k)$. В корреляторах мономов будем производить сокрашения, пользуясь соотношениями (20)-(22). Полученные $\delta$-функции 
будем передвигать между $b_{i}^{\epsilon}(t, k)$, пользуясь соотношениями $(23),(24)$. Будем продолжать эту процедуру, пока не приведем моном к нормально упорядоченному виду. Поскольку функционал $\langle\langle\cdot\rangle\rangle$ есть вакуумное среднее, то в коррелятор войдет вклад только от $\delta$-функций.

Спаривания $b_{1}\left(t_{m_{h}^{\prime}}, k_{m_{h}^{\prime}}\right) b_{1}^{\dagger}\left(t_{m_{h}}, k_{m_{h}}\right)$ и $b_{2}\left(t_{m_{h}}, k_{m_{h}}\right) b_{2}^{\dagger}\left(t_{m_{h}^{\prime}}, k_{m_{h}^{\prime}}\right)$ по соотношениям (20), (21) равны

$$
\delta\left(k_{m_{h}^{\prime}}-k_{m_{h}}\right) c_{m_{h} m_{h}^{\prime}}\left(k_{m_{h}}\right) 2 \pi \delta\left(t_{m_{h}^{\prime}}-t_{m_{h}}\right) \delta\left(\widetilde{\omega}\left(k_{m_{h}}\right)+k_{m_{h}} p-\varepsilon_{h} k_{m_{h}}^{2}\right) .
$$

Соотношения (23), (24) дают вклад

$$
\sum_{\alpha}(-1)^{\varepsilon_{\alpha}} \chi_{\left(m_{\alpha}, m_{\alpha}^{\prime}\right)}\left(m_{h}\right)
$$

в фазовый сдвиг (аргумент последней $\delta$-функции в (25)), возникаюший при переносе $\delta$-функций через $b_{i}^{\epsilon}(t, k)$. Это завершает доказательство теоремы 2.

Поля $b_{i}$ из температурной свободной алгебры возникают как стохастический предел конструкции Араки-Вудса. Изложим эту конструкцию в бозонном варианте. Конструкция Араки-Вудса позволяет представить температурное состояние бозона через вакуумное состояние пары бозонов.

Введем два независимых бозонных поля $c_{1}(k), c_{2}(k)$,

$$
\left[c_{i}(k), c_{j}^{\dagger}\left(k^{\prime}\right)\right]=\delta_{i j} \delta\left(k-k^{\prime}\right) .
$$

Каждое из полей $c_{i}(k)$ действует в фоковском представлении. Рассмотрим преобразование Боголюбова

$$
\begin{aligned}
a(k) & =\sqrt{m(k)} c_{1}(k)+\sqrt{m(k)-1} c_{2}^{\dagger}(k), \\
a^{\dagger}(k) & =\sqrt{m(k)} c_{1}^{\dagger}(k)+\sqrt{m(k)-1} c_{2}(k) .
\end{aligned}
$$

Тогда

$$
\left[a(k), a^{\dagger}\left(k^{\prime}\right)\right]=\delta\left(k-k^{\prime}\right) .
$$

Для вакуумных средних имеем соотношение

$$
\left\langle a(k) a^{\dagger}\left(k^{\prime}\right)\right\rangle=m(k) \delta\left(k-k^{\prime}\right) .
$$

Если взять

$$
m(k)=\frac{1}{1-e^{-\beta \omega_{k}}},
$$

то получим температурное состояние.

В стохастическом пределе оператор $a_{\lambda}(t, k)$ перейдет в сумму двух белых шумов $b_{1}(t, k)+b_{2}^{\dagger}(t, k)$. Формула (26) примет вид

$$
\begin{aligned}
b(t, k)= & \lim _{\lambda \rightarrow 0} \frac{1}{\lambda} e^{-i \frac{t}{\lambda^{2}}(\widetilde{\omega}(k)+k p)} e^{-i k q} a(k)= \\
= & \lim _{\lambda \rightarrow 0} \frac{1}{\lambda} e^{-i \frac{t}{\lambda^{2}}(\widetilde{\omega}(k)+k p)} e^{-i k q} \sqrt{m(k)} c_{1}(k)+ \\
& \quad+\lim _{\lambda \rightarrow 0} \frac{1}{\lambda} e^{-i \frac{t}{\lambda^{2}}(\widetilde{\omega}(k)+k p)} e^{-i k q} \sqrt{m(k)-1} c_{2}^{\dagger}(k)= \\
= & b_{1}(t, k)+b_{2}^{\dagger}(t, k) .
\end{aligned}
$$




\section{5. ВЫЧИСЛЕНИЕ $n$-ТОЧЕЧНОГО КОРРЕЛЯТОРА}

В этом разделе будут вычислены стохастические пределы $n$-точечных корреляторов для модели взаимодействия частищы с нерелятивистским квантовым полем, т.е. доказана теорема 1.

Используя правила перемножения вейлевских операторов

$$
e^{i \alpha p} e^{i \beta q}=e^{i(\alpha p+\beta q)} e^{i \frac{1}{2} \alpha \beta}
$$

получим для свободной эволюции полевого оператора (7) выражение

$$
a_{\lambda}^{\epsilon}(t, k) \equiv \frac{1}{\lambda} \exp \left[-i(-1)^{\epsilon}\left\{\frac{t}{\lambda^{2}}(\widetilde{\omega}(k)+k p)+k q-\frac{1}{2} \frac{t}{\lambda^{2}} k^{2}\right\}\right] a^{\epsilon}(k) .
$$

При последовательности индексов $\varepsilon=\left\{\epsilon_{2 n}, \ldots, \epsilon_{1}\right\} \in\{1,0\}^{2 n}$ получим, что

$$
\begin{aligned}
\left\langle\prod_{j=1}^{2 n} a_{\lambda}^{\epsilon_{j}}\left(t_{j}, k_{j}\right)\right\rangle= & \prod_{j=1}^{2 n}\left\{\frac{1}{\lambda} \exp \left[-i(-1)^{\epsilon_{j}}\left\{\frac{t_{j}}{\lambda^{2}}\left(\widetilde{\omega}\left(k_{j}\right)+k_{j} p\right)+k_{j} q-\frac{1}{2} \frac{t_{j}}{\lambda^{2}} k_{j}^{2}\right\}\right]\right\} \times \\
& \times\left\langle\prod_{h=1}^{2 n} a^{\epsilon_{h}}\left(k_{h}\right)\right\rangle
\end{aligned}
$$

причем

$$
\left\langle\prod_{h=1}^{2 n} a^{\epsilon_{h}}\left(k_{h}\right)\right\rangle=\sum_{m_{h}^{\prime} \neq m_{h}} \prod_{h=1}^{n} \delta\left(k_{m_{h}^{\prime}}-k_{m_{h}}\right) c_{m_{h} m_{h}^{\prime}}\left(k_{m_{h}}\right),
$$

что равняется сумме спариваний всех операторов рождения-уничтожения. Операторы в этих произведениях считаются упорядоченными справа налево. Поэтому можно записать

$$
\begin{aligned}
\left\langle\prod_{j=1}^{2 n} a_{\lambda}^{\epsilon_{j}}\left(t_{j}, k_{j}\right)\right\rangle= & \prod_{j=1}^{2 n}\left\{\frac{1}{\lambda} \exp \left[-i(-1)^{\epsilon_{j}}\left\{\frac{t_{j}}{\lambda^{2}}\left(\widetilde{\omega}\left(k_{j}\right)+k_{j} p\right)+k_{j} q-\frac{1}{2} \frac{t_{j}}{\lambda^{2}} k_{j}^{2}\right\}\right]\right\} \times \\
& \times \sum_{m_{h}^{\prime} \neq m_{h}} \prod_{h=1}^{n} \delta\left(k_{m_{h}^{\prime}}-k_{m_{h}}\right) c_{m_{h} m_{h}^{\prime}}\left(k_{m_{h}}\right) .
\end{aligned}
$$

Используя правила перемножения вейлевских операторов, видим, что

$$
\begin{aligned}
& \prod_{j=1}^{2 n}\left\{\frac{1}{\lambda} \exp \left[-i(-1)^{\epsilon_{j}}\left\{\frac{t_{j}}{\lambda^{2}}\left(\widetilde{\omega}\left(k_{j}\right)+k_{j} p\right)+k_{j} q-\frac{1}{2} \frac{t_{j}}{\lambda^{2}} k_{j}^{2}\right\}\right]\right\}= \\
& =\exp \left\{-\frac{i}{2} \sum_{1 \leqslant j<l \leqslant 2 n}(-1)^{\epsilon_{j}+\epsilon_{l}} k_{j} k_{l} \frac{t_{j}-t_{l}}{\lambda^{2}}\right\} \times \\
& \quad \times\left(\frac{1}{\lambda}\right)^{2 n} \exp \left[-i \sum_{j=1}^{2 n}(-1)^{\epsilon_{j}}\left\{\frac{t_{j}}{\lambda^{2}}\left(\widetilde{\omega}\left(k_{j}\right)+k_{j} p\right)+k_{j} q-\frac{1}{2} \frac{t_{j}}{\lambda^{2}} k_{j}^{2}\right\}\right] .
\end{aligned}
$$


Используя то, что $m^{\prime}{ }_{h}$ пробегает половину $2 n$ индексов $l$ и $m_{h}$ пробегает другую половину этих индексов, а также то, что $(-1)^{\epsilon_{m^{\prime}}}{ }_{h}=1$ и $(-1)^{\epsilon_{m}}=-1$, получаем для фазового фактора, входяшего в формулу (32), следуюшее выражение:

$$
\begin{aligned}
& -\frac{i}{2} \sum_{l=1}^{2 n} \sum_{j<l}(-1)^{\epsilon_{j}+\epsilon_{l}} k_{j} k_{l}\left(t_{j}-t_{l}\right)= \\
& =-\frac{i}{2} \sum_{h=1}^{n}\left\{\sum_{1 \leqslant j<m^{\prime}{ }_{h}}(-1)^{\epsilon_{j}} k_{j} k_{m^{\prime}{ }_{h}}\left(t_{j}-t_{m^{\prime}{ }_{h}}\right)-\sum_{1 \leqslant j<m_{h}}(-1)^{\epsilon_{j}} k_{j} k_{m_{h}}\left(t_{j}-t_{m_{h}}\right)\right\}= \\
& =-\frac{i}{2} \sum_{h=1}^{n}\left\{\sum_{\alpha}^{m^{\prime}{ }_{\alpha}<m^{\prime}{ }_{h}} k_{m^{\prime}{ }_{\alpha}} k_{m^{\prime}{ }_{h}}\left(t_{m^{\prime} \alpha}-t_{m^{\prime}{ }_{h}}\right)-\sum_{\beta}^{m_{\beta}<m_{h}^{\prime}} k_{m_{\beta}} k_{m^{\prime}{ }_{h}}\left(t_{m_{\beta}}-t_{m_{h}{ }_{h}}\right)-\right. \\
& \left.-\sum_{\gamma}^{m^{\prime} \gamma<m_{h}} k_{m_{\gamma}^{\prime}} k_{m_{h}}\left(t_{m^{\prime}{ }^{\prime}}-t_{m_{h}}\right)+\sum_{\delta}^{m_{\delta}<m_{h}} k_{m_{\delta}} k_{m_{h}}\left(t_{m_{\delta}}-t_{m_{h}}\right)\right\} \equiv \\
& \equiv-\frac{i}{2} \sum_{h=1}^{n}\left(\mathrm{I}_{h}+\mathrm{II}_{h}\right)
\end{aligned}
$$

Здесь учитьвается, что $k_{m_{h}}=k_{m_{h}}$. Группируя первый член с третьим и второй с четвертым, получим

$$
\begin{aligned}
& \mathrm{I}_{h}=\sum_{\alpha}^{m^{\prime}{ }_{\alpha}<m^{\prime}{ }_{h}} k_{m_{\alpha}} k_{m_{h}}\left(t_{m^{\prime}{ }_{\alpha}}-t_{m_{h}^{\prime}{ }_{h}}\right)-\sum_{\gamma}^{m^{\prime}{ }_{\gamma}<m_{h}} k_{m_{\gamma}} k_{m_{h}}\left(t_{m^{\prime}{ }_{\gamma}}-t_{m_{h}}\right)= \\
& =\sum_{\alpha}^{m^{\prime}{ }_{\alpha}<m^{\prime}{ }_{h}} k_{m_{\alpha}} k_{m_{h}}\left(t_{m^{\prime}{ }_{\alpha}}-t_{m_{h}}\right)+\sum_{\alpha}^{m^{\prime}{ }_{\alpha}<m^{\prime}{ }_{h}} k_{m_{\alpha}} k_{m_{h}}\left(t_{m_{h}}-t_{m_{h}{ }_{h}}\right)- \\
& -\sum_{\gamma}^{m_{\gamma}^{\prime}<m_{h}} k_{m_{\gamma}} k_{m_{h}}\left(t_{m^{\prime}{ }_{\gamma}}-t_{m_{h}}\right)=\sum_{\alpha}^{m_{h}<m_{\alpha}^{\prime}<m_{h}^{\prime}} k_{m_{\alpha}} k_{m_{h}}\left(t_{m^{\prime}{ }_{\alpha}}-t_{m_{h}}\right)+ \\
& +\sum_{\alpha}^{m^{\prime}{ }_{\alpha}<m_{h}^{\prime}} k_{m_{\alpha}} k_{m_{h}}\left(t_{m_{h}}-t_{m_{h}{ }_{h}}\right) \text { при } m_{h}^{\prime}>m_{h}
\end{aligned}
$$

и

$$
\begin{aligned}
\mathrm{I}_{h}= & -\sum_{\alpha}^{m_{h}^{\prime}<m_{\alpha}^{\prime}<m_{h}} k_{m_{\alpha}} k_{m_{h}}\left(t_{m^{\prime}{ }_{\alpha}}-t_{m_{h}}\right)+ \\
& +\sum_{\alpha}^{m^{\prime}{ }_{\alpha}<m^{\prime}{ }_{h}} k_{m_{\alpha}} k_{m_{h}}\left(t_{m_{h}}-t_{m^{\prime}{ }_{h}}\right)+k_{m_{h}} k_{m_{h}}\left(t_{m_{h}}-t_{m_{h}^{\prime}}\right) \text { при } m^{\prime}{ }_{h}<m_{h}
\end{aligned}
$$

Для суммы второго и четвертого членов получим

$$
-\mathrm{II}_{h}=\sum_{\beta}^{m_{\beta}<m^{\prime}{ }_{h}} k_{m_{\beta}} k_{m_{h}}\left(t_{m_{\beta}}-t_{m_{h}{ }^{\prime}}\right)-\sum_{\delta}^{m_{\delta}<m_{h}} k_{m_{\delta}} k_{m_{h}}\left(t_{m_{\delta}}-t_{m_{h}}\right)=
$$




$$
\begin{aligned}
= & \sum_{\beta}^{m_{\beta}<m^{\prime}{ }_{h}} k_{m_{\beta}} k_{m_{h}}\left(t_{m_{\beta}}-t_{m^{\prime}{ }_{h}}\right)-\sum_{\delta}^{m_{\delta}<m_{h}} k_{m_{\delta}} k_{m_{h}}\left(t_{m_{\delta}}-t_{m_{h}^{\prime}}\right)- \\
& -\sum_{\delta}^{m_{\delta}<m_{h}} k_{m_{\delta}} k_{m_{h}}\left(t_{m^{\prime}{ }_{h}}-t_{m_{h}}\right)=\sum_{\beta}^{m_{h}<m_{\beta}<m^{\prime}{ }_{h}} k_{m_{\beta}} k_{m_{h}}\left(t_{m_{\beta}}-t_{m^{\prime}{ }_{h}}\right)+ \\
& +\sum_{\delta}^{m_{\delta}<m_{h}} k_{m_{\delta}} k_{m_{h}}\left(t_{m_{h}}-t_{m^{\prime}{ }_{h}}\right)+k_{m_{h}} k_{m_{h}}\left(t_{m_{h}}-t_{m^{\prime}{ }_{h}}\right) \text { при } m^{\prime}{ }_{h}>m_{h}
\end{aligned}
$$

и

$$
\begin{aligned}
-\mathrm{II}_{h}= & -\sum_{\beta}^{m_{h}^{\prime}<m_{\beta}<m_{h}} k_{m_{\beta}} k_{m_{h}}\left(t_{m_{\beta}}-t_{m^{\prime}{ }_{h}}\right)+ \\
& +\sum_{\delta}^{m_{\delta}<m_{h}} k_{m_{\delta}} k_{m_{h}}\left(t_{m_{h}}-t_{{m^{\prime}}_{h}}\right) \text { при } m^{\prime}{ }_{h}<m_{h} .
\end{aligned}
$$

Теперь можно записать, что

$$
\begin{aligned}
& \mathrm{I}_{h}+\mathrm{II}_{h}=\sum_{\alpha}^{m_{h}<m_{\alpha}^{\prime}<m_{h}^{\prime}} k_{m_{\alpha}} k_{m_{h}}\left(t_{m_{\alpha}^{\prime}}-t_{m_{h}}\right)+\sum_{\alpha}^{m^{\prime}{ }_{\alpha}<m_{h}^{\prime}} k_{m_{\alpha}} k_{m_{h}}\left(t_{m_{h}}-t_{m^{\prime}{ }_{h}}\right)- \\
& -\sum_{\beta}^{m_{h}<m_{\beta}<m_{h}^{\prime}} k_{m_{\beta}} k_{m_{h}}\left(t_{m_{\beta}}-t_{m^{\prime}}\right)-\sum_{\beta}^{m_{\beta}<m_{h}} k_{m_{\beta}} k_{m_{h}}\left(t_{m_{h}}-t_{m_{h}^{\prime}}\right)- \\
& -k_{m_{h}} k_{m_{h}}\left(t_{m_{h}}-t_{m_{h}{ }_{h}}\right) \text { при } m_{h}^{\prime}>m_{h}
\end{aligned}
$$

и

$$
\begin{aligned}
\mathrm{I}_{h}+\mathrm{II}_{h}= & -\sum_{\alpha}^{m_{h}^{\prime}<m_{\alpha}^{\prime}<m_{h}} k_{m_{\alpha}} k_{m_{h}}\left(t_{m^{\prime} \alpha}-t_{m_{h}}\right)+\sum_{\alpha}^{m^{\prime}{ }_{\alpha}<m^{\prime}{ }_{h}} k_{m_{\alpha}} k_{m_{h}}\left(t_{m_{h}}-t_{m^{\prime}}\right)+ \\
& +\sum_{\beta}^{m_{h}^{\prime}<m_{\beta}<m_{h}} k_{m_{\beta}} k_{m_{h}}\left(t_{m_{\beta}}-t_{m^{\prime}{ }_{h}}\right)-\sum_{\beta}^{m_{\beta}<m_{h}} k_{m_{\beta}} k_{m_{h}}\left(t_{m_{h}}-t_{m^{\prime}{ }_{h}}\right)+ \\
& +k_{m_{h}} k_{m_{h}}\left(t_{m_{h}}-t_{m_{h}^{\prime}}\right) \text { при } m_{h}^{\prime}<m_{h} .
\end{aligned}
$$

Рассмотрим в формуле (32) следуюший член:

$$
\begin{aligned}
\left(\frac{1}{\lambda}\right)^{2 n} & \exp \left[-i \sum_{j=1}^{2 n}(-1)^{\epsilon_{j}}\left\{\frac{t_{j}}{\lambda^{2}}\left(\widetilde{\omega}\left(k_{j}\right)+k_{j} p\right)+k_{j} q-\frac{1}{2} \frac{t_{j}}{\lambda^{2}} k_{j}^{2}\right\}\right] \times \\
& \times \sum_{m_{h}^{\prime} \neq m_{h}} \prod_{h=1}^{n} \delta\left(k_{m_{h}^{\prime}}-k_{m_{h}}\right) c_{m_{h} m_{h}^{\prime}}\left(k_{m_{h}}\right) .
\end{aligned}
$$


Заметим, что

$$
\begin{aligned}
\sum_{1 \leqslant l \leqslant 2 n}(-1)^{\epsilon_{l}} t_{l} k_{l} & =-\sum_{1 \leqslant h \leqslant n}\left(t_{m_{h}}-t_{m^{\prime}{ }_{h}}\right) k_{m_{h}}, \\
\sum_{1 \leqslant l \leqslant 2 n}(-1)^{\epsilon_{l}} k_{l} q & =0 .
\end{aligned}
$$

Тогда рассматриваемый член принимает вид

$$
\begin{aligned}
\left(\frac{1}{\lambda}\right)^{2 n} & \exp \left[i \sum_{1 \leqslant h \leqslant n} \frac{t_{m_{h}}-t_{m_{h}^{\prime}}}{\lambda^{2}}\left(\widetilde{\omega}\left(k_{m_{h}}\right)+k_{m_{h}} p-\frac{1}{2} k_{m_{h}}^{2}\right)\right] \times \\
& \times \sum_{m_{h}^{\prime} \neq m_{h}} \prod_{h=1}^{n} \delta\left(k_{m_{h}^{\prime}}-k_{m_{h}}\right) c_{m_{h} m_{h}^{\prime}}\left(k_{m_{h}}\right) .
\end{aligned}
$$

Сделаем замену переменных

$$
\left\{\begin{array}{l}
u_{m_{h}}=t_{m_{h}} \\
v_{m_{h}}=t_{m_{h}}-t_{m_{h}^{\prime}} .
\end{array}\right.
$$

Приведенные выше выкладки позволяют сформулировать следуюшую лемму.

Лемма 1. Для рассматриваемого в теореме 1 коррелятора имеет место формула

$$
\begin{aligned}
\left\langle\prod_{j=1}^{2 n} a_{\lambda}^{\epsilon_{j}}\left(t_{j}, k_{j}\right)\right\rangle=\exp & {\left[-\frac{i}{2} \frac{1}{\lambda^{2}} \sum_{h=1}^{n}\left\{\mathrm{I}_{h}+\mathrm{II}_{h}\right\}\right] \times } \\
& \times\left(\frac{1}{\lambda}\right)^{2 n} \exp \left[i \sum_{1 \leqslant h \leqslant n} \frac{v_{m_{h}}}{\lambda^{2}}\left(\widetilde{\omega}\left(k_{m_{h}}\right)+k_{m_{h}} p-\frac{1}{2} k_{m_{h}}^{2}\right)\right] \times \\
& \times \sum_{m_{h}^{\prime} \neq m_{h}} \prod_{h=1}^{n} \delta\left(k_{m_{h}^{\prime}}-k_{m_{h}}\right) c_{m_{h} m_{h}^{\prime}}\left(k_{m_{h}}\right)
\end{aligned}
$$

Фазовий фактор в (37) равен

$$
\begin{aligned}
& \sum_{\alpha}^{m_{h}<m^{\prime}{ }_{\alpha}<m^{\prime}{ }_{h}} k_{m_{\alpha}} k_{m_{h}}\left(-v_{m_{\alpha}}+u_{m_{\alpha}}-u_{m_{h}}\right)+\sum_{\alpha}^{m^{\prime}{ }_{\alpha}<m^{\prime}{ }_{h}} k_{m_{\alpha}} k_{m_{h}} v_{m_{h}}- \\
& \quad-\sum_{\beta}^{m_{h}<m_{\beta}<m^{\prime}{ }_{h}} k_{m_{\beta}} k_{m_{h}}\left(v_{m_{h}}+u_{m_{\beta}}-u_{m_{h}}\right)- \\
& -\sum_{\beta}^{m_{\beta}<m_{h}} k_{m_{\beta}} k_{m_{h}} v_{m_{h}}-k_{m_{h}} k_{m_{h}} v_{m_{h}} \quad n p u \quad m_{h}^{\prime}>m_{h}
\end{aligned}
$$


$u$

$$
\begin{aligned}
- & \sum_{\alpha}^{m_{h}^{\prime}<m_{\alpha}^{\prime}<m_{h}} k_{m_{\alpha}} k_{m_{h}}\left(-v_{m_{\alpha}}+u_{m_{\alpha}}-u_{m_{h}}\right)+\sum_{\alpha}^{m_{\alpha}^{\prime}<m^{\prime}{ }_{h}} k_{m_{\alpha}} k_{m_{h}} v_{m_{h}}+ \\
& +\sum_{\beta}^{m_{h}^{\prime}<m_{\beta}<m_{h}} k_{m_{\beta}} k_{m_{h}}\left(v_{m_{h}}+u_{m_{\beta}}-u_{m_{h}}\right)- \\
& -\sum_{\beta}^{m_{\beta}<m_{h}} k_{m_{\beta}} k_{m_{h}} v_{m_{h}}+k_{m_{h}} k_{m_{h}} v_{m_{h}} \quad \text { npu } \quad m^{\prime}{ }_{h}<m_{h} .
\end{aligned}
$$

По лемме Римана-Лебега осцилляторные множители типа $\exp \left(i k^{2} u / \lambda^{2}\right)$ исчезают в пределе $\lambda \rightarrow 0$. Таким образом, в этом пределе разбиение $\left\{\left(m_{h}, m_{h}^{\prime}\right)\right\}$ в (8) "выживает" тогда и только тогда, когда для любого фиксированного $h=1, \ldots, n$ и для любого $\alpha$ или

$$
m_{h}<m_{\alpha}<m_{h}^{\prime} \Leftrightarrow m_{h}<m_{\alpha}^{\prime}<m_{h}^{\prime},
$$

или

$$
m_{h}>m_{\alpha}>m_{h}^{\prime} \Leftrightarrow m_{h}>m_{\alpha}^{\prime}>m_{h}^{\prime},
$$

т.е. имеет место разбиение, соответствуюшее непересекаюшейся полуплоской диаграмме. Соответственно только нетривиальные разбиения последовательности $\varepsilon=\left\{\epsilon_{2 n}, \ldots\right.$ $\left.\ldots, \epsilon_{1}\right\} \in\{1,0\}^{2 n}$ дают нетривиальный вклад в предел коррелятора. Обозначая через $\left\{\left(m_{h}, m_{h}^{\prime}\right)\right\}$ разбиение, видим, что соответствуюшее значение фазового фактора (38) равно

$$
\begin{gathered}
\sum_{\alpha}^{m_{h}<m_{\alpha}^{\prime}<m^{\prime}{ }_{h}} k_{m_{\alpha}} k_{m_{h}}\left(-v_{m_{\alpha}}-v_{m_{h}}\right)+\sum_{\alpha}^{m_{\alpha}<m^{\prime}{ }_{h}} k_{m_{\alpha}} k_{m_{h}} v_{m_{h}}- \\
-\sum_{\beta}^{m_{\beta}<m_{h}} k_{m_{\beta}} k_{m_{h}} v_{m_{h}}-k_{m_{h}} k_{m_{h}} v_{m_{h}} \text { при } m_{h}^{\prime}>m_{h}
\end{gathered}
$$

и

$$
\begin{gathered}
-\sum_{\alpha}^{m_{h}^{\prime}<m_{\alpha}^{\prime}<m_{h}} k_{m_{\alpha}} k_{m_{h}}\left(-v_{m_{\alpha}}-v_{m_{h}}\right)+\sum_{\alpha}^{m_{\alpha}^{\prime}<m^{\prime}{ }_{h}} k_{m_{\alpha}} k_{m_{h}} v_{m_{h}}- \\
-\sum_{\beta}^{m_{\beta}<m_{h}} k_{m_{\beta}} k_{m_{h}} v_{m_{h}}+k_{m_{h}} k_{m_{h}} v_{m_{h}} \text { при } m_{h}^{\prime}<m_{h} .
\end{gathered}
$$

Исследуем полученный фазовый фактор. Для $m_{h}^{\prime}>m_{h}$ имеем

$$
\sum_{\alpha}^{m^{\prime}{ }_{\alpha}<m^{\prime}{ }_{h}} k_{m_{\alpha}} k_{m_{h}} v_{m_{h}}=\sum_{\alpha}^{m_{h}<m^{\prime}{ }_{\alpha}<m^{\prime}{ }_{h}} k_{m_{\alpha}} k_{m_{h}} v_{m_{h}}+\sum_{\alpha}^{m^{\prime}{ }_{\alpha} \leqslant m_{h}} k_{m_{\alpha}} k_{m_{h}} v_{m_{h}} .
$$


Так как $m^{\prime}{ }_{\alpha} \neq m_{h}$, то

$$
\sum_{\alpha}^{m^{\prime}{ }_{\alpha} \leqslant m_{h}} k_{m_{\alpha}} k_{m_{h}} v_{m_{h}}=\sum_{\alpha}^{m^{\prime}{ }_{\alpha}<m_{h}} k_{m_{\alpha}} k_{m_{h}} v_{m_{h}}
$$

Следовательно, фазовый фактор равен

$$
\begin{aligned}
& -\sum_{\alpha}^{m_{h}<m_{\alpha}^{\prime}<m_{h}^{\prime}} k_{m_{\alpha}} k_{m_{h}} v_{m_{\alpha}}+\sum_{\alpha}^{m_{\alpha}^{\prime}<m_{h}} k_{m_{\alpha}} k_{m_{h}} v_{m_{h}}- \\
& \quad-\sum_{\beta}^{m_{\beta}<m_{h}} k_{m_{\beta}} k_{m_{h}} v_{m_{h}}-k_{m_{h}} k_{m_{h}} v_{m_{h}}
\end{aligned}
$$

В случае $m^{\prime}{ }_{h}<m_{h}$ из условия отсутствия пересечений получаем, что

$$
-\sum_{\alpha}^{m_{h}^{\prime}<m^{\prime}{ }_{\alpha}<m_{h}} k_{m_{\alpha}} k_{m_{h}}\left(-v_{m_{\alpha}}-v_{m_{h}}\right)=-\sum_{\alpha}^{m_{h}^{\prime}<m_{\alpha}<m_{h}} k_{m_{\alpha}} k_{m_{h}}\left(-v_{m_{\alpha}}-v_{m_{h}}\right) .
$$

Таким образом, фазовый фактор равен

$$
\begin{aligned}
& \sum_{\alpha}^{m_{h}^{\prime}<m^{\prime}{ }_{\alpha}<m_{h}} k_{m_{\alpha}} k_{m_{h}} v_{m_{\alpha}}+\sum_{\alpha}^{m^{\prime}{ }_{\alpha}<m^{\prime}{ }_{h}} k_{m_{\alpha}} k_{m_{h}} v_{m_{h}}- \\
& \quad-\sum_{\beta}^{m_{\beta}<m_{h}^{\prime}} k_{m_{\beta}} k_{m_{h}} v_{m_{h}}+k_{m_{h}} k_{m_{h}} v_{m_{h}} .
\end{aligned}
$$

Введем обозначение

$$
\mathrm{I}_{h}+\mathrm{II}_{h}=\Phi_{h}-(-1)^{\varepsilon_{h}} k_{m_{h}} k_{m_{h}} v_{m_{h}} .
$$

Здесь $(-1)^{\varepsilon_{h}}=1$ для $m_{h}^{\prime}>m_{h}$ и $(-1)^{\varepsilon_{h}}=-1$ для $m_{h}^{\prime}<m_{h}$. Получаем для фазового фактора формулы

$$
\begin{aligned}
\Phi_{h}= & -\sum_{\alpha \in\left(m_{h}, m_{h}^{\prime}\right) \text { или }\left(m_{h}^{\prime}, m_{h}\right)}(-1)^{\varepsilon_{h}} k_{m_{\alpha}} k_{m_{h}} v_{m_{\alpha}}- \\
& -\sum_{\alpha: h \in\left(m_{\alpha}, m_{\alpha}^{\prime}\right) \text { или }\left(m_{\alpha}^{\prime}, m_{\alpha}\right)}(-1)^{\varepsilon_{\alpha}} k_{m_{\alpha}} k_{m_{h}} v_{m_{h}}, \\
\sum_{1 \leqslant h \leqslant n} \Phi_{h}= & -2 \sum_{1 \leqslant h \leqslant n}(-1)^{\varepsilon_{\alpha}} k_{m_{\alpha}} k_{m_{h}} v_{m_{h}}= \\
= & -2 \sum_{1 \leqslant h \leqslant n} \sum_{\alpha}(-1)^{\varepsilon_{\alpha}} \chi_{\left(m_{\alpha}, m_{\alpha}^{\prime}\right)}\left(m_{h}\right) k_{m_{\alpha}} k_{m_{h}} v_{m_{h}} .
\end{aligned}
$$

Здесь $\chi_{\left(m_{\alpha}, m^{\prime}{ }_{\alpha}\right)}$ есть индикатор интервала $\left(m_{\alpha}, m^{\prime}{ }_{\alpha}\right)$ или $\left(m^{\prime}{ }_{\alpha}, m_{\alpha}\right)$. Таким образом, доказана следующая лемма. 
ЛЕмма 2. Вклад в коррелятор от непересекающихся диаграмм равен

$$
\begin{gathered}
\left(\frac{1}{\lambda}\right)^{2 n} \exp \left[i \sum _ { 1 \leqslant h \leqslant n } \frac { v _ { m _ { h } } } { \lambda ^ { 2 } } \left(\left(\widetilde{\omega}\left(k_{m_{h}}\right)+k_{m_{h}} p\right)+\sum_{\alpha}(-1)^{\varepsilon_{\alpha}} \chi_{\left(m_{\alpha}, m_{\alpha}^{\prime}\right)}\left(m_{h}\right) k_{m_{\alpha}} k_{m_{h}}-\right.\right. \\
\left.\left.-\frac{1}{2} k_{m_{h}}^{2}+\frac{1}{2}(-1)^{\varepsilon_{h}} k_{m_{h}}^{2}\right)\right] \sum_{m_{h}^{\prime} \neq m_{h}} \prod_{h=1}^{n} \delta\left(k_{m_{h}^{\prime}}-k_{m_{h}}\right) c_{m_{h} m_{h}^{\prime}}\left(k_{m_{h}}\right) .
\end{gathered}
$$

Используя формулу (11) и удерживая только нетривиальные разбиения, получим, что

$$
\begin{aligned}
\lim _{\lambda \rightarrow 0} & \left\langle a_{\lambda}^{\epsilon_{2 n}}\left(t_{2 n}, k_{2 n}\right) a_{\lambda}^{\epsilon_{2 n-1}}\left(t_{2 n-1}, k_{2 n-1}\right) \ldots a_{\lambda}^{\epsilon_{1}}\left(t_{1}, k_{1}\right)\right\rangle= \\
= & \sum_{m_{h}^{\prime} \neq m_{h}} \prod_{h=1}^{n} \delta\left(k_{m_{h}^{\prime}}-k_{m_{h}}\right) c_{m_{h} m_{h}^{\prime}}\left(k_{m_{h}}\right) 2 \pi \delta\left(t_{m_{h}^{\prime}}-t_{m_{h}}\right) \times \\
& \quad \times \delta\left(\widetilde{\omega}\left(k_{m_{h}}\right)+k_{m_{h}} p+\sum_{\alpha}(-1)^{\varepsilon_{\alpha}} \chi_{\left(m_{\alpha}, m^{\prime}{ }_{\alpha}\right)}\left(m_{h}\right) k_{m_{\alpha}} k_{m_{h}}-\varepsilon_{h} k_{m_{h}}^{2}\right),
\end{aligned}
$$

где $\left\{\left(m_{j}^{\prime}, m_{j}\right): j=1, \ldots, n\right\}$ есть нетривиальное разбиение $\{1, \ldots, 2 n\}$, ассоциированное $\mathrm{c} \varepsilon$. Теорема 1 доказана.

Работа выполнена при частичной поддержке гранта INTAS, № 96-0698 и гранта РФФИ, № 96-01-00312.

\section{Список литературы}

[1] Н. Н. Боголюбов. УМЖ. 1950. Т. 2. № 2. С. 3-24.

[2] H. Fröhlich. Adv. in Phys. 1954. V. 3. P. 325.

[3] С. Швебер. Введение в релятивистскую квантовую теорию поля. М.: ИЛ, 1963.

[4] Р. Фейнман. Статистическая механика. М.: Мир, 1975.

[5] L. Accardi, A. Frigerio, Y. G. Lu. Commun. Math. Phys. 1990. V. 131. P. 537-570.

[6] L. Accardi, S. Kozyrev, I. V. Volovich. Phys. Rev. A. 1997. V. 56. P. 375.

[7] L. Accardi, Y. G. Lu. Commun. Math. Phys. 1996. V. 180. № 3. P. 605-632.

[8] M. Skeide. Hilbert modules in quantum electrodynamics and quantum probability: Preprint Universitat Heidelberg, 1996.

[9] J. Gough. J. of Phys. A. 1997. V. 149. P. 2375.

[10] J. Cuntz. Commun. Math. Phys. 1977. V. 57. P. 173-185.

[11] D. E. Evans. Publ. Res. Inst. Math. Sci. 1980. V. 16. P. 915-927.

[12] H. Araki, D. E. Evans. J. Operat. Theory. 1981. V. 23. P. 326.

[13] D. Voiculescu, K. J. Dykema, A. Nica. Free random variables. CRM Monograph Series. V.1. Amer. Math. Soc., 1992.

[14] B. Kummerer, R. Speicher. J. Funct. Anal. 1992. V. 103. P. 372-478.

[15] O. Bratteli, P.E.T. Jorgensen, A. Kishimoto, R.F. Werner. Pure states on $O_{d}$. func-an/9711004.

[16] I. Ya. Aref'eva, I. V. Volovich. Nucl. Phys. B. 1996. V. 462. P. 600.

Поступила в редакцию 17.II.1998 г., после доработки 28.V.1998 г. 